\title{
Enhancement of Odontoblastic Differentiation of Stem Cells from Exfoliated Deciduous Tooth Using N-acetylcysteine- An In Vitro Study
}

\author{
Madhura Pawar ${ }^{1}$, Vivek Pawar ${ }^{2}$, Sanjay G Thete ${ }^{3}$, Smita D Dutta ${ }^{4}$, Pramod P Sadan ${ }^{5}$, Rahul Maria ${ }^{6}$, Dinraj Kulkarni ${ }^{7}$
}

\begin{abstract}
Aim and objective: The study was conducted to evaluate the effects of $\mathrm{N}$-acetylcysteine (NAC) on the propagation and differentiation of stem cells from human exfoliated deciduous teeth(SHED).

Materials and methods: SHEDs were isolated by explant culture method and characterized for stem cell properties using flow cytometry method. MTT assay and Cell Counting Kit-8 (CCK-8) assay were used to examine the viability and proliferation of the SHEDs. The effects of NAC-induced osteo/odontoblastic differentiation of SHEDs were determined by functional staining for mineralization, and the gene expression of osteo/odontoblastic transcription factors and proteins was evaluated by real-time quantitative reverse transcription-polymerase chain reaction (qRT-PCR) analyses. Protein levels of collagen type 1 (COL1), dentin sialophosphoprotein (DSPP), and dentin matrix acidic phosphoprotein 1 (DMP-1) were calculated by the Western blot method to assess the osteo/odontogenic differentiation.

Results: SHEDs presented mesenchymal stem cell (MSC)-like characteristics on flow cytometric analysis. The cell viability and metabolic activity of SHEDs were increased with an increase in the concentrations of NAC from 0.5 to $10 \mathrm{nM}$. However, the concentrations of NAC from 0.5 to $2.5 \mathrm{mM}$ did not affect cell proliferation. NAC incorporated at a concentration of $2.5 \mathrm{mM}$ showed higher mineralization and considerably increased gene expression levels of runt-related transcription factor 2 (RUNX2), COL1A1, DSPP, and DMP-1. It significantly increased the protein expression of odontoblast-related matrix proteins like COL1, DSPP, and DMP-1.

Conclusion: NAC regulates the healthy propagation of dental stem cells in vitro. Its effects on the differentiation of dental pulp SHEDs remain unidentified. This study explores that NAC can encourage the mineralization of SHEDs and differentiate them into the odontoblastic lineage.

Clinical significance: The results propose that NAC could have a significant pharmacological role in activating and enhancing odontogenic differentiation of dental stem cells and possibly a prospect in regenerative dentistry.

Keywords: DMP-1, DSPP, N-acetylcysteine, Odontogenic differentiation, Stem cells.

The Journal of Contemporary Dental Practice (2021): 10.5005/jp-journals-10024-3140
\end{abstract}

\section{INTRODUCTION}

The multipotent stromal cells known as mesenchymal stem cells (MSCs) possess the proficiency to give rise to the cell types of several tissues..$^{1-3}$ These multipotent cells can be obtained from various tissue sources, including bone marrow, umbilical cord blood, dental pulp, and adipose tissues. Studies have shown that these cells have the potential to differentiate into multiple specialized cell lineages both in vitro and in vivo conditions and hence proven advantageous for cellular therapeutic replacement. ${ }^{4,5}$ Stem cells from dental sources are of a certain type of MSCs and can be acquired from tooth pulp, ligament of periodontium, tooth follicle, and apical papilla. ${ }^{1,6}$ Individually, these tissues yield a diverse type of stem cell. ${ }^{1,2,6}$ Stem cells from dental pulp (DPSCs), together with stem cells from the exfoliated deciduous tooth (SHEDs), have the aptitude for self-renewing capacity and differentiation into multiple tissue cell types, particularly to differentiate into osteoblasts, odontoblasts, hepatocytes, neural cells, adipocytes, myocytes, and chondrocytes. ${ }^{78}$ SHEDs exhibit expression of a definite set of cell surface markers that are solitary found in embryonic stem cells and MSCs, comprising CD73, CD90, CD105, CD106, STRO 1, SOX2, NANOG, OCT4, and CMyc. ${ }^{2,9,10}$ When compared with DPSCs or other dental stem cells, SHEDs have demonstrated a superior rate of proliferation than others; also in mice models, SHEDs have shown a great capability for the formation of dentin/bone. ${ }^{1,2,11}$
${ }^{1}$ Department of Pedodontics and Preventive Dentistry, Dr DY Patil Dental College and Hospital, Dr DY Patil Vidyapeeth, Pune, Maharashtra, India

${ }^{2}$ Department of Oral and Maxillofacial Surgery, SMBT Institute of Dental Sciences, Nashik, Maharashtra, India

${ }^{3}$ Department of Oral Pathology and Microbiology, Rural Dental College, PIMS (DU), Loni, Maharashtra, India

${ }^{4}$ Department of Conservative Dental Sciences and Endodontics, College of Dentistry, Quassim University, Buraydah, Al-Quassim, Saudi Arabia

${ }^{5}$ Department of Prosthetic Dental Sciences, Quassim University, Buraydah, Al-Quassim, Saudi Arabia

${ }^{6}$ Department of Conservative Dental Sciences and Endodontics, Bhabha Dental College, Bhopal, Madhya Pradesh, India

${ }^{7}$ Department of Oral Pathology and Microbiology, MA Rangoonwala College of Dental Sciences and Research Center, Pune, Maharashtra, India

Corresponding Author: Madhura Pawar, Department of Pedodontics and Preventive Dentistry, Dr DY Patil Dental College and Hospital, Dr DY Patil Vidyapeeth, Pune, Maharashtra, India, Phone: +91 9860079769, e-mail:drmadhura4@gmail.com

How to cite this article: Pawar M, Pawar V, Thete SG, et al. Enhancement of Odontoblastic Differentiation of Stem Cells from Exfoliated Deciduous Tooth Using N-acetylcysteine-An In Vitro Study. J Contemp Dent Pract 2021;22(8):882-889.

Source of support: Nil

Conflict of interest: None

(0) The Author(s). 2021 Open Access This article is distributed under the terms of the Creative Commons Attribution 4.0 International License (https://creativecommons. org/licenses/by-nc/4.0/), which permits unrestricted use, distribution, and non-commercial reproduction in any medium, provided you give appropriate credit to the original author(s) and the source, provide a link to the Creative Commons license, and indicate if changes were made. The Creative Commons Public Domain Dedication waiver (http://creativecommons.org/publicdomain/zero/1.0/) applies to the data made available in this article, unless otherwise stated. 
Oral and dental sciences, including periodontics, endodontics, prosthodontics, and orthodontics, are arriving at an innovative age by using the multipotent stem cells and bioactive materials as a tool for the reparation and regeneration of the dental and oral tissues. ${ }^{12,13}$ Several types of stem cells are being investigated for this perseverance. ${ }^{12,14}$ However, the lower cell survival rate during differentiation of stem cells and after transplantation of welldifferentiated stem cells at the ischemic or damaged tissue sites may be due to the microenvironment that is not favorable to the viability of these cells. ${ }^{15,16}$

The continuous oxidative stress during the cell differentiation process leads to the production of reactive oxygen species (ROS) that are a regular derivative of the usual energy metabolism. ${ }^{17,18}$ ROS are found to perform a crucial part in the development of stem cells and homeostasis of the tissues as lesser ROS generation results in excessive proliferation rate, higher rate of cell survival, and enhanced differentiation of stem cells, while too much ROS generation might lead to the dysfunction of the mitochondrial, higher cellular death, inflammation in the tissues, and stem cell aging by possibly affecting their key characteristics of regeneration and differentiation. ${ }^{17,19-21}$

$\mathrm{N}$-acetylcysteine (NAC) is a glutathione (GSH) precursor and a potent antioxidant that is effective in both direct and indirect ways to reduce the production of intracellular oxidative stress and in turn free radical production.

The direct action of NAC is by interacting with the electrophilic groups of free radicals through its free thiol sidechain.

The indirect antioxidant action of NAC is through the replenishment of intracellular $\mathrm{GSH}$, the body's major antioxidant. Considering the massive antioxidant potential of GSH and lower concentrations of NAC inside cells, the predominant antioxidant effects of NAC may be associated with maintaining GSH levels in the intracellular environment.

Previous studies have demonstrated that the treatment of NAC promotes cell proliferation in MSCs, and these NAC-treated MSCs retained their properties that stem cells possess and their differentiation potential. ${ }^{22,23}$

Hence, the study was conducted to assess the effects of NAC on the proliferative potential and osteo/odontoblastic differentiation ability of SHEDs. The expression of dentin sialophosphoprotein (DSPP) and dentin matrix acidic phosphoprotein 1 (DMP-1) proteins in NAC-mediated odontoblastic differentiation was also investigated.

\section{Materials and Methods}

\section{Sample Collection}

After the approval from the institutional ethics committee of Dr DY Patil Dental College and Hospital, Pimpri, Pune (Reference no. RM 15/19), informed consent was obtained from the participants of the study. Non-carious human exfoliated deciduous teeth were obtained from healthy participants aged $6-12$ years $(n=10)$ after taking informed consent. The pulp was extracted in sterilized conditions and transferred to the laboratory directly for further processing.

\section{Isolation and Culture of SHEDs}

Isolation of SHEDs was carried out using the explant culture method. Briefly, the tissue was cut into small fragments, and those pieces were employed in culture flasks with complete culture media [Dulbecco's modified eagle medium (DMEM) with a supplementation of $20 \%$ fetal bovine serum (FBS)]. The dishes were subjected to incubation at $37^{\circ} \mathrm{C}$ in a humid incubator with $5 \% \mathrm{CO}_{2}$. The complete nutritive medium was replaced with fresh media after every 2 days, and the outgrowth of the cells was checked frequently under a phase-contrast microscope, an inverted type (Olympus CKX53, Japan). The outstrip cells with $>80 \%$ conflux was removed using $0.25 \%$ trypsin-EDTA solution and expanded further. The cell could differentiate till passage 4 , and the cells differentiated at passage 4 were used during the further procedures. ${ }^{24}$

\section{Colony-forming Unit (CFU) Assay for the Clonogenic Ability of SHEDs}

To evaluate the clonogenic ability of SHEDs to form colonies from single cells, a CFU assay was done. Cells were inoculated onto 6-well cell culture plates at $5 \times 10^{2}$ per well in culture media (DMEM with $10 \%$ FBS). Subsequently 7 days of incubation, the wells were stained with $0.3 \%$ of crystal violet. ${ }^{24}$

\section{Population Doubling Time and Growth Curve for SHEDs}

For the determination of the proliferative capability of SHEDs, $1 \times 10^{4}$ cells at passage 4 from individual samples were inoculated into 12 well cell culture dishes, and on every alternate day, the cells were counted for 14 days. The growth curve for 14 days was compassed using cell numbers only. ${ }^{24}$

\section{Characterization of SHEDs Using Flow Cytometry}

SHEDs grown on the surface of the flask were detached and washed with phosphate-buffered saline (PBS). After incubation for 30 minutes with STRO-1, CD106, CD34, and HLA-DR (all from eBiosciences, USA) antibodies, the cells were again washed with PBS and the cells were analyzed on Attune NxT Flow Cytometer (Thermo Scientific, USA). No less than 10,000 events were acquired for each sample. The degree of positive staining was considered as a percentage comparing to the isotype control. The cell differentiation potential was evaluated in three replicates for each lineage. ${ }^{24}$

\section{Differentiation}

\section{Adipocyte Differentiation}

For differentiation into adipocytes, the SHEDs were exposed to an adipogenic induction medium and incubated for 21 days. The medium was changed twice per week. To determine the adipogenic differentiation, after 21 days, the cells were fixed with $4 \%$ paraformaldehyde followed by staining for lipid droplets with $0.3 \%$ oil red $0 .{ }^{24}$

\section{Osteoblast Differentiation}

For osteoblastic differentiation, the SHEDs were exposed to an osteogenic induction medium and incubated for 21 days. The medium was changed twice per week. To determine the osteogenic differentiation, after 21 days, the cells were fixed with $4 \%$ paraformaldehyde followed by staining for mineralization with $2 \%$ Alizarin Red S (pH, 4.1-4.3). ${ }^{24}$

\section{Chondrocyte Differentiation}

To differentiate into chondrocytes, the SHEDs were exposed to a chondrogenic induction medium and incubated for four weeks. 
The medium was changed every alternate day. To determine the chondrogenic differentiation, after 4 weeks, the cells were fixed with $4 \%$ paraformaldehyde followed by staining for glycosaminoglycans using $0.1 \%$ alcian blue. ${ }^{24}$

\section{3-(4,5-Dimethylthiazol-2-yl)-2,5-diphenyltetrazolium Bromide (MTT) Assay}

The viability of the SHEDs was assessed by using an MTT assay. The cells were seeded into 96 -well plates at $5 \times 10^{3}$ cells per well with $100 \mu \mathrm{L}$ of MSC Basal Medium + supplements (ALLCELLS, USA) and incubated for 24 hours at $37^{\circ} \mathrm{C}, 5 \% \mathrm{CO}_{2}$. The medium was then replaced with $200 \mu \mathrm{L}$ of fresh medium containing different concentrations of NAC $(0.5,1,2.5$, and $5 \mathrm{mM})$. Untreated cells were used as the control group. Two sets of 96 well culture plates were prepared for each of the NAC concentrations, and each of the two sets was incubated for 48 hours at $37^{\circ} \mathrm{C}, 5 \% \mathrm{CO}_{2}$.

Following this, the $20 \mu \mathrm{L}$ of MTT solution at $0.5 \mathrm{mg} / \mathrm{mL}$ concentration was added to the individual well and incubated for4 hours at $37^{\circ} \mathrm{C}, 5 \% \mathrm{CO}_{2}$. Subsequently, the media was replaced with $100 \mu \mathrm{L}$ dimethyl sulfoxide in every well. The absorbance was taken at $570 \mathrm{~nm}$ on a spectrophotometer (Sigma-Aldrich, Germany). ${ }^{25}$

\section{Cell Proliferation Assays}

The evaluation of proliferation of SHEDs was done by using a Cell Counting Kit-8 (CCK-8) (Sigma-Aldrich, St. Louis, Missouri, USA) at $24,48,72$ hours, and 7 th day as described previously. ${ }^{15}$ CCK-8 is a colorimetric reaction-based assay that yields an orange formazan dye to an extent that is directly proportional to the number of cells. The cells at passage 3 of different concentrations of $\operatorname{NAC}(0.5,1$, 2.5 , and $5 \mathrm{mM}$ ) and untreated cells as control group were seeded into 96-well plates with minimum essential medium eagle-alpha modification (a-MEM) at a density of $2 \times 10^{3}$ cells per well (two replicates per group). Ten microliters of CCK-8 reagent (Dojindo, Shanghai, China) was added to each well at 24, 48, 72 hours, and 7 th day, and the cells were incubated at $37^{\circ} \mathrm{C}$.The proliferation rate of SHEDs was calculated by the absorbance taken at $450 \mathrm{~nm}$ on a spectrophotometer (Sigma-Aldrich, Germany). ${ }^{25}$

\section{Osteogenic/Odontogenic Differentiation}

The cells at passage 3 of different concentrations of NAC (0.5, $1,2.5$, and $5 \mathrm{mM}$ ) and untreated cells as control group were exposed to an osteogenic induction medium composed of a-MEM supplemented with $10 \% \mathrm{FBS}, 100 \mathrm{IU} / \mathrm{mL}$ penicillin, $100 \mu \mathrm{g} / \mathrm{mL}$ streptomycin, $50 \mu \mathrm{g} / \mathrm{mL}$ L-ascorbic acid, $10 \mathrm{mM}$ $\beta$-glycerophosphate, $10 \mathrm{nM}$ calcitriol (1a,25-dihydroxyvitamin D3), and $10 \mathrm{nM}$ dexamethasone (all from Sigma-Aldrich, USA). The cells were incubated for 21 days, and the fresh media were replaced every alternate day during this period.

To determine the osteogenic mineralization, after 21 days, the cells were fixed with $4 \%$ paraformaldehyde followed by staining for mineralization with $2 \%$ Alizarin Red S (pH, 4.1-4.3). The quantitation of Alizarin Red S-stained osteoblasts was done by dissolving stained cells in $4 \% \mathrm{NaOH}$, and the dissolved stain was read spectrophotometrically at $450 \mathrm{~nm}$ (Sigma-Aldrich, Germany). ${ }^{25}$

\section{Real-time PCR for the Analysis of Gene Expression}

Total RNA was isolated from the cells of different concentrations of NAC $(0.5,1,2.5$, and $5 \mathrm{mM})$ and untreated cells as control group by using the TRIzol Reagent (Invitrogen, Thermo Fisher Scientific, USA).
Table1: List of primers

\begin{tabular}{lll}
\hline Gene & \multicolumn{1}{c}{ Forward primer } & \multicolumn{1}{c}{ Reverse primer } \\
\hline RUNX2 & $5^{\prime}$-GAC TGT GGT TAC CGT & $5^{\prime}$-ACT TGG TTT TTC ATA \\
& CAT GGC-3' & ACA GCG GA-3' \\
COL1A1 & $5^{\prime}$-GAG GGC CAA GAC & $5^{\prime}$-CAG ATC ACG TCA TCG \\
& GAA GAC ATC-3' & CAC AAC-3' \\
OCN & $5^{\prime}$-CTC ACA CTC GCC CTA & $5^{\prime}$-TTG GAC ACA AAG GCT \\
& TT-3' & GCA C-3' \\
DSPP & $5^{\prime}$-TGG AGC CAC AAA CAG & $5^{\prime}$-TCC AGC TAC TTG AGG \\
& AAG CAA-3' & TCC ATC-3' \\
DMP-1 & $5^{\prime}$-GTG AGT GAG TCC AGG & $5^{\prime}$-TTT TGA GTG GGA GAG \\
& GGA GAT AA-3' & TGT GTG C-3' \\
ACTB & $5^{\prime}$-AGA GCT ACG AGC TGC & $5^{\prime}$-AGC ACT GTG TTG GCG \\
& CTG AC-3' & TAC AG-3' \\
\hline
\end{tabular}

One microgram RNA was transcribed reversely by using the TAKARA Reverse Transcriptase kit (TAKARA, Osaka, Japan) conferring to the guidelines by the manufacturer. Quantitative analysis of genes of interest [runt-related transcription factor 2 (RUNX2), collagen type 1 (COL1), osteocalcin (OCN), DSPP, and DMP-1] was done by using the SYBR Green PCR Master Mix on a real-time quantitative reverse transcription-polymerase chain reaction(qRT-PCR) system. Normalization of expressions of target genes to $ß$-actin was done by using the $\Delta \Delta \mathrm{Ct}$ technique. The quantification of data was done by using the $2-\Delta \Delta \mathrm{Ct}$ technique and shown as normalized relative gene expression to that of the average $\mathrm{Ct}$ for the $\beta$-actin gene. The list of the primers is in Table 1. Experiments were performed in triplicate for each sample. ${ }^{25,26}$

\section{Western Blot Analysis}

The cells of different concentrations of $\operatorname{NAC}(0.5,1,2.5$, and $5 \mathrm{mM})$ and untreated cells as control group were lysed using lysis buffer, and the lysates were centrifuged at $12,000 \mathrm{rpm}$ for 15 minutes. The supernatant was collected, and the protein content was determined using the protein assay. SDS-PAGE sample buffer was introduced to the lysates, and the lysates were heated to $100^{\circ} \mathrm{C}$ for 8 minutes. Of the total protein, $20 \mathrm{mg}$ was loaded in each well of a $10 \%$ SDS-PAGE gel (Bio-Rad, Munich, Germany). Immunoreactive proteins were seen by using the ECLkit (Beyotime Biotech, Shanghai, China). The gray value of protein bands was calculated by image J software. ${ }^{25}$

\section{Statistical Analysis}

All the data values were showed by the way of mean \pm standard deviation. Altogether, the respective experimental groups were compared with a one-way analysis of variance test. $p$-value $<0.05$ and $* * p<0.01$.

\section{Results \\ Morphological Characteristics and Proliferation of SHEDs}

The morphological features of SHEDs isolated by the explant culture technique were monitored under an inverted phase-contrast microscope. SHEDs showed elongated spindle-shaped morphology (Fig. 1A). Moreover, SHEDs demonstrated a good colony-forming (clonogenic) ability (Fig. 1B) and $10 \pm 1$ hours of population doubling time at passage 4 indicating its homogeneity (Fig. 1C). 

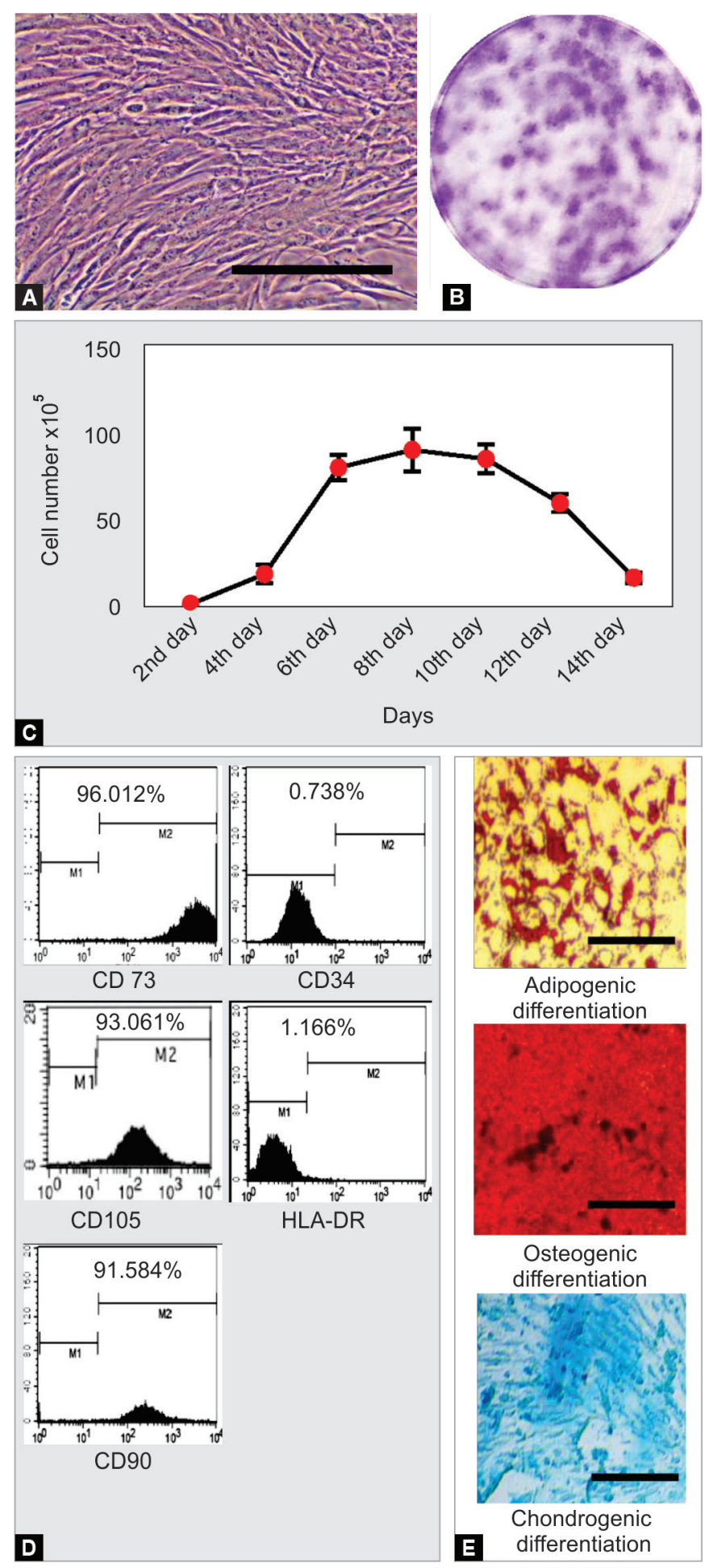

Figs $1 \mathrm{~A}$ to E: Isolation and characterization of SHEDs. (A) SHEDs at passage 4; (B) DPSCs grew on monolayer and made CFU-fibroblast; (C) Growth curve analysis of DPSCs at passage 4; (D) Analysis by flow cytometry of SHEDs for MSCs-specific cell surface markers; (E) Differentiation of SHEDs into adipocytes, osteoblasts, and chondrocytes $(n=5)$. Scale bar $=100 \mu \mathrm{m}$

\section{Mesenchymal Stem Cell-specific Surface Markers}

SHEDs expressed STRO-1 and CD106, which are MSC-specific surface markers (Fig. 1B); however, the expression of CD34 (hematopoietic lineage markers) and HLA-DR (MHC class-Il cell surface receptor) was observed to be negative in all cultured SHEDs (Fig. 1D).

\section{In Vitro Differentiation into Adipogenic, Osteogenic, and Chondrogenic Lineages}

Furthermore, the SHEDs from all the samples showed the ability to differentiate into adipocytes, osteoblasts, and chondrocytes (Fig. 1E), which is a characteristic property of multipotent MSCs (Patil et al. ${ }^{24}$ ).

\section{Treatment of SHEDs with Different Concentrations of NAC}

We treated SHEDs with different concentrations of NAC, and it was evident that after 48 hours of treatment, a decrease in the cell metabolites was observed by MTT assay (Fig. 2A). However, a slightly higher dose of $1.0 \mathrm{mM}$ showed a significant increase in cell metabolism. The cell proliferation was found to be slower at a higher concentration of NAC ( $5 \mathrm{mM}$ ) after 48 hours (Fig. 2B).

In our investigation, we assessed mineralization after osteogenic induction with and without NAC (Fig. 2C). We observed that the mineralization was more prominent and significantly higher in the induced SHEDs treated with $1.0 \mathrm{mM}$ concentration of NAC (Fig. 2D).

In this investigation, mRNA levels of RUNX2, OCN, DSPP, and DMP-1 were found to be upregulated in SHEDs induced with $1.0 \mathrm{mM}$ NAC concentration. Conversely, there was no outcome observed by NAC on the mRNA level of COL1 during the late stage of the treatment (Fig. 3). However, remains unexplained the lower expression of COL1 in the NAC-induced odontoblasts.

The protein expression of COL1, DSPP, and DMP-1 in the SHEDs induced with different concentrations of NAC $(0.5,1,2.5$, and $5 \mathrm{mM}$ ) and untreated cells as control group were assessed, and the result showed the higher levels of protein expression of COL1, DSPP, and DMP-1 in the SHEDs induced with NAC at $1.0 \mathrm{mM}$ specified the progression of odontogenesis of the SHEDs (Fig. 4).

The results of our investigation show that the effect of NAC on SHEDs is dose-dependent. Also, the concentration of $1.0 \mathrm{mM}$ NAC showed higher cell proliferation and enhanced osteogenic potential of cultured cells.

\section{Discussion}

For its uses in clinical therapeutic fields, a source of MSCs must be easily available and accessible with high content of totipotent stem cells. Dental pulp tissue attains all these criteria, hence preferred for stem cell regenerative therapy. $5,24,26$

Cell therapies originate from biological sources, such as MSCs. This biological seed material is then manipulated in a laboratory to develop the cell therapy product. Despite their clinical and commercial promise, novel cellular therapies are nascent technologies that have not yet achieved widespread clinical utility and commercial viability. Stem cells demonstrate lower cell survival and amplified rate of cellular death either during cell differentiation in a laboratory or after transplantation at the ischemic or damaged tissue sites.Unavoidable production of ROS owing to continuous oxidative stress throughout the differentiation process and in ischemic or injured tissues is thought to be the main hurdle for the success of stem cell therapy. NAC being a precursor of biological antioxidant, GSH can function as a strong inhibitor of ROS. ${ }^{27,28}$ Its effects on the differentiation of dental pulp SHEDs remain 

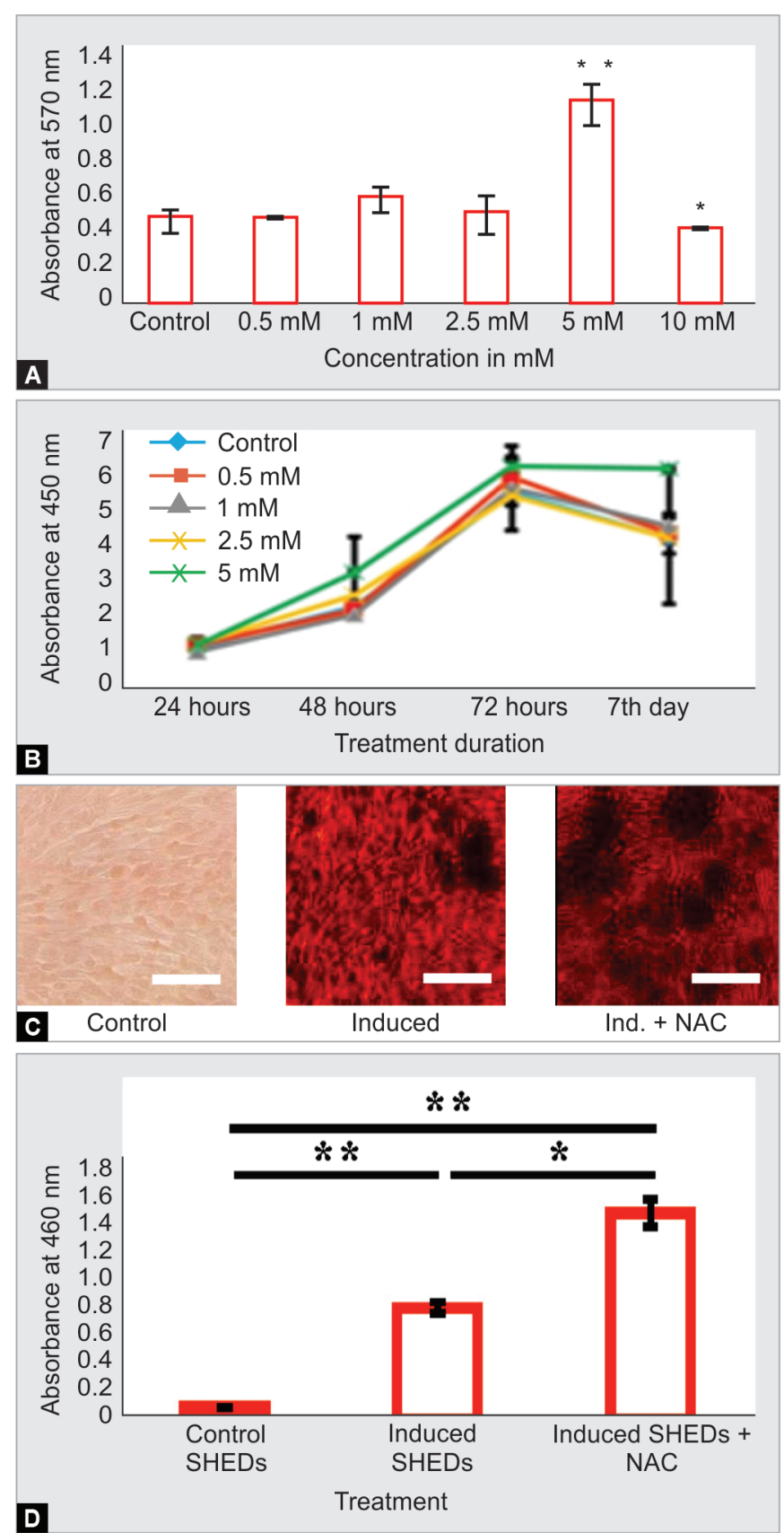

Figs $2 A$ to D: Effect of NAC on the cell viability, proliferation, and mineralized nodule formation in SHEDs. (A) MTT assay for the viability of SHEDs after NAC treatment; (B) Effect of NAC on the proliferation of SHEDs; (C) SHEDs were cultured in an osteogenic induction medium for 21 days with and without the presence of NAC $(2.5 \mathrm{mM})$; cells were retreated every 2 days; mineralization was assessed by Alizarin Red $\mathrm{S}$ staining (scale bar $=100 \mathrm{~mm}$ ); (D) On the 21 st day, the calcium content was determined $(n=5)$. n.s., not significant, $* p<0.05$, $* * p<0.01$. NAC, $\mathrm{N}$-acetylcysteine

unidentified. Hence, the investigation was intended to study the effects of NAC on the propagation and differentiation of SHEDs.

In our study, the explant culture method was used for the isolation of cells from SHED. The explant culture method was used over the enzymatic digestion to avoid any chemical or mechanical stress and the use of bacterial and xeno-derived products for clinical utility for the isolation of cells. ${ }^{24}$

Upon isolation by explant culture, the cells showed a resemblance to MSC-like morphological characteristics and MSC-specific cell surface markers, an ideal source of MSCs for clinical cellular therapeutic applications. The result demonstrated that SHEDs bear a resemblance to MSC-like morphological characteristics and MSC-specific cell surface markers.

NAC has therapeutic effects over a range of disorders like chronic obstructive pulmonary disease, chronic bronchitis, cystic fibrosis, heavy metal toxicity, and psychiatric/neurological disorders. Being an $\mathrm{N}$-acetyl derivative of the amino acid L-cysteine, NAC is a GSH precursor that helps scavenge free radicals and bind metal ions into complexes. ${ }^{27}$ Since NAC possesses anti-inflammatory activity by inhibition of nuclear factor kappa-light-chain-enhancer of activated $B$ cells, it has been used for regulating oxidative stress and inflammation-related diseases. ${ }^{28,29}$

This property of regulating oxidative stress of NAC was used in our study. With the aim to thwart cellular death caused by oxidative stress during cellular differentiation, the cells were treated with various concentrations of NAC. The addition of NAC has shown to modify the progression of the cell cycle MSCs by downregulation of inhibitors of CDK, and with the introduction of NAC, the cells showed enhanced proliferation rate, hitherto reserved their stemness properties and the differentiation potential. ${ }^{30}$ The results of our study showed that NAC does not affect the viability and proliferation in SHEDs at lower concentrations of $0.1 \mathrm{mM}$.

Being totipotent cells, SHEDs can differentiate into odontoblast lineages when a suitable induction medium is provided. ${ }^{25,26}$ Odontoblasts cause dentinogenesis in which the formation of dentin takes place in response to the synthesis of organic matrix and the deposition of subsequent mineralized crystals in the matrix. ${ }^{31,32}$ This arrangement of dentin formation is alike to that of the bone, which is also a mineralized connective tissue. ${ }^{25}$ In our investigation, NAC-induced osteogenic differentiation in SHEDs shows enhanced mineralization.

RUNX2, an important transcription factor, and master regulator control the formation and development of bone and teeth by the regulation of totipotent MSC differentiation into odontoblastic lineages. ${ }^{33}$ The upregulation of RUNX2 in our study confirmed the odontoblastic differentiation process when treated with NAC.

DSPP, which is synthesized highly by the odontoblastic cells, is necessary for the development and calcification of the dentin matrix. ${ }^{34}$ Research has revealed that DSPP is associated with the mineralization of reparation of dentin as well as bone. ${ }^{9,10}$ Hence,DSPP remains a foremost characteristic marker for odontogenic differentiation. Also, studies have shown the expression of DMP-1 during earlier osteogenic

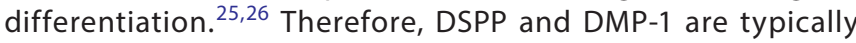
designated as definite protein markers of odontogenic differentiation to identify the odontogenic differentiation capability of SHEDs. In our investigation, SHEDs induced with different concentrations of $\operatorname{NAC}(0.5,1,2.5$, and $5 \mathrm{mM})$ had different effects on cultured cells. In other words, it can be stated the effect of NAC on SHEDs is dosedependent. Also, the concentration of 1.0 mM NAC showed higher cell proliferation and enhanced osteogenic potential of cultured cells. At $1.0 \mathrm{mM}$ concentration, NAC triggered odontoblastic differentiation by elevating the protein levels of DSPP and DMP-1. The observed result could be attributed to decreased levels of oxidative stress in cell cultures at $1.0 \mathrm{mM}$ concentration of NAC. 

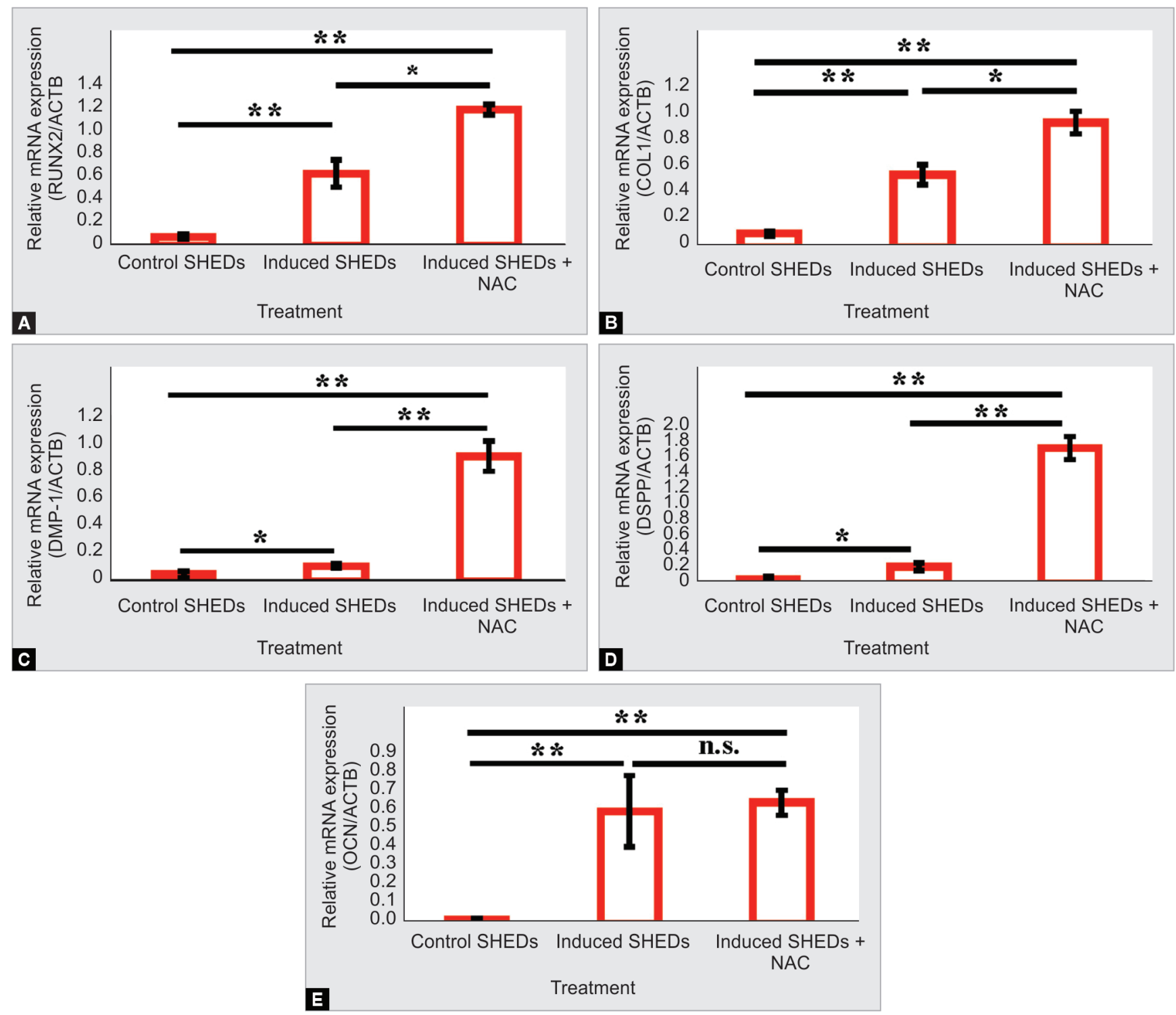

Figs $3 A$ to E: Gene expression analysis of odontoblast-related genes in SHEDs. The analysis of mRNA expression of RUNX2, COL1, DSPP, DMP-1, and OCN was carried out using qRT-PCR $(n=5)$. n.s., not significant, $* p<0.05, * * p<0.01$. NAC, N-acetylcysteine

Previously published results also demonstrated that NAC in concentrations of 0.1 and $1.0 \mathrm{mM}$ increased the proliferation of MSCs to the DTSCs culture at $21 \% \mathrm{O}_{2}$, thereby enhancing their number diminishing oxidative damage, changing enzymes activity, and priming cells for osteo-chondrogenesis. ${ }^{22}$

Stem cells from tooth pulp have proven their significance for utilization in a plethora of clinical uses. It is imperative to investigate the factors that can modulate the biological behavior of SHEDs.

This study enlightens the precedence of NAC for the usage of SHEDs for research and clinical use. Therefore, we presented the distinctive use of NAC as an efficient, reproducible, and biological antioxidant of clinical utility, which may be preferred for obtaining human dental pulp tissue-derived stem cells for tissue engineering and regenerative medicine.

Analysis of previously published research underlines the important role of NAC and the level of free oxygen radicals in the differentiation process. ${ }^{22}$ Although a conclusion can be drawn from our investigation that the effect of NAC on SHEDs is dose-dependent, the possible underlining mechanism of its effect is unknown. Also, the other limitation of the study is that we cannot elucidate the reason for the negative effects of higher concentrations (2.5 and $5 \mathrm{mM}$ ) of NAC on induced SHEDs.

\section{ConClusion}

In summary, to the best of our knowledge, this is the first study revealing the pharmacological role of NAC in activating and enhancing odontogenic differentiation of dental stem cells increasing the expressions of the DSPP and DMP-1 proteins.

Although the specific role and mechanism of NAC in the odontogenic differentiation of SHEDs require further investigation, our findings extended the current knowledge on the pharmacological role of NAC in the odontogenic differentiation 


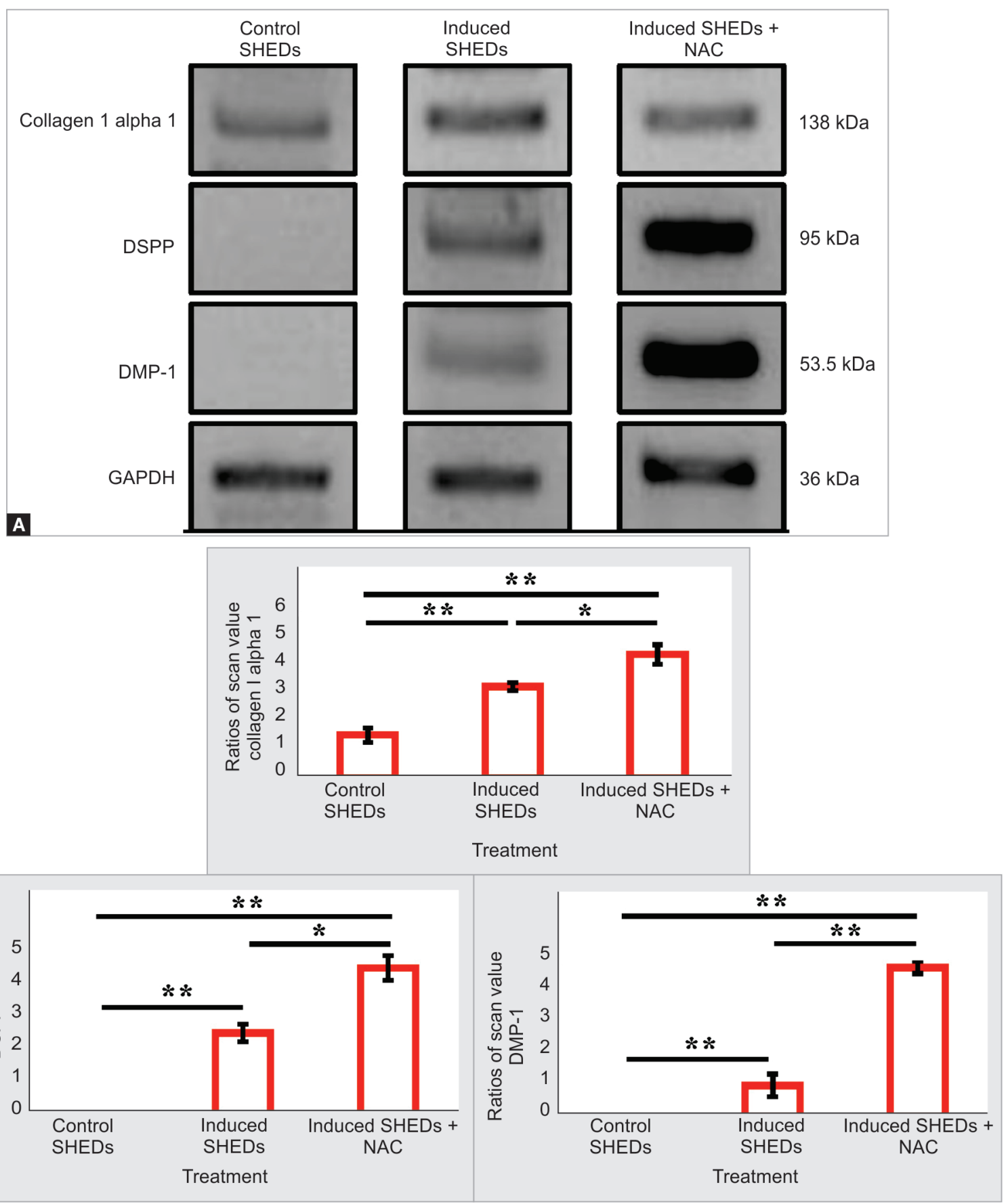

Figs 4A and B: NAC upregulates protein expression of DSPP and DMP-1 in SHEDs. (A) Whole-cell extracts were evaluated by using the Western blot method to decide the protein expression levels of DSPP, DMP-1, and COL1 in SHEDs; (B) The graphs show the ratios of band densities of DSPP, DMP-1, and COL1 ( $n=5)$. n.s., not significant, $* p<0.05, * * p<0.01$. NAC, N-acetylcysteine

process of SHEDs and suggested additional in vivo experimentation implemented utilizing novel animal models to validate the prospect of impending clinical utility as well as amplify treatments for the regeneration and repair of the dentin-pulp complex.

\section{References}

1. Zhang N, Chen B, Wang W, et al. Isolation, characterization, and multilineage differentiation of stem cells from human exfoliated deciduous teeth. Mol Med Rep 2016;14(1):95-102. DOI: 10.3892/mmr.2016.5214.
2. Zhu Q, Gao J, Tian G, et al. Adrenomedullin promotes the odontogenic differentiation of dental pulp stem cells through CREB/BMP2 signaling pathway. Acta Biochemic Biophys Sin 2017;49(7):609-616. DOI: $10.1093 / \mathrm{abbs} / \mathrm{gm} \times 053$.

3. Garzon I, Martin-Piedra MA, Carriel V, et al. Bioactive injectable aggregates with nanofibrous microspheres and human dental pulp stem cells: a translational strategy in dental endodontics. J Tissue Eng Regen Med 2018;12(1):204-216. DOI: 10.1002/term.2397.

4. Abuarqoub D, Awidi A, Abuharfeil N. Comparison of osteo/ odontogenic differentiation of human adult dental pulp stem cells and stem cells from apical papilla in the presence of 
platelet lysate. Arch Oral Biol2015;60(10):1545-1553. DOI: 10.1016/ j.archoralbio.2015.07.007.

5. Tangtrongsup S, Kisiday JD. Differential effects of the antioxidants $\mathrm{N}$-acetylcysteine and pyrrolidine dithiocarbamate on mesenchymal stem cell chondrogenesis. Cell Mol Bioeng 2019;12(2):153-163. DOI: 10.1007/s12195-019-00566-3.

6. Martacic J, Filipovic MK, Borozan S, et al. N-acetyl-I-cysteine protects dental tissue stem cells against oxidative stress in vitro. Clin Oral Invest 2018;22(8):2897-2903. DOI: 10.1007/s00784-018-2377-2.

7. Zhang D, Li Q, Rao L, et al. Effect of 5-Aza-2'-deoxycytidine on odontogenic differentiation of human dental pulp cells. J Endod 2015;41(5):640-645. DOI: 10.1016/j.joen.2014.12.006.

8. Zeng L, Zhao N, Han D, et al. DLX3 mutation negatively regulates odontogenic differentiation of human dental pulp cells. Arch Oral Biol 2017;77:12-17. DOI: 10.1016/j.archoralbio.2017.01.011.

9. Zhou M, Liu NX, Shi SR, et al. Effect of tetrahedral DNA nanostructures on proliferation and osteo/odontogenic differentiation of dental pulp stem cells via activation of the notch signaling pathway. Nanomedicine 2018;14(4):1227-1236. DOI: 10.1016/j.nano.2018.02.004.

10. Zou T, Dissanayaka WL, Jiang S, et al. Semaphorin 4D enhances angiogenic potential and suppresses osteo-/odontogenic differentiation of human dental pulp stem cells. J Endod 2017;43(2):297-305. DOI: 10.1016/j.joen.2016.10.019.

11. Heng BC, Ye $X$, Liu $Y$, et al. Effects of recombinant overexpression of $\mathrm{BCL} 2$ on the proliferation, apoptosis, and osteogenic/ odontogenic differentiation potential of dental pulp stem cells. J Endod 2016;42(4):575-583. DOI: 10.1016/j.joen.2016.01.013.

12. Heng BC, Wang S, Gong T, et al. Ephrin B2 signalling enhances osteogenic/odontogenic differentiation of human dental pulp stem cells. Arch Oral Biol 2018;87:62-71. DOI: 10.1016/ j.archoralbio.2017.12.014.

13. Liu M, Zhao L, Hu J, et al. Endothelial cells, and endothelin-1 promote the odontogenic differentiation of dental pulp stem cells. Mol Med Rep 2018;18(1):893-901. DOI: 10.3892/mmr.2018.9033.

14. Sonoda S, Mei YF, Atsuta I, et al. Exogenous nitric oxide stimulates the odontogenic differentiation of rat dental pulp stem cells. Sci Rep 2018;8(1):3419. DOI: 10.1038/s41598-018-21183-6.

15. Ching HS, Luddin N, Rahman IA, et al. Expression of odontogenic and osteogenic markers in DPSCs and SHED: a review. Curr Stem Cell Res Ther 2017;12(1):71-79. DOI: 10.2174/1574888x11666160815 095733.

16. Rahimi S, Salarinasab S, Ghasemi N, et al. In vitro induction of odontogenic activity of human dental pulp stem cells by white Portland cement enriched with zirconium oxide and zinc oxide components. J Dent Res Dent Clin Dent Prospects 2019;13(1):3-10. DOI: 10.15171/joddd.2019.001.

17. Teti G, Salvatore V, Focaroli S, et al. In vitro osteogenic and odontogenic differentiation of human dental pulp stem cells seeded on carboxymethyl cellulose-hydroxyapatite hybrid hydrogel. Front Physiol 2015;6:1-10. DOI: 10.3389/fphys.2015.00297.

18. Lian M, Zhang $Y$, Shen Q, et al. JAB1 accelerates odontogenic differentiation of dental pulp stem cells. J Mol Histol 2016;47(3): 317-324. DOI: 10.1007/s10735-016-9672-5.

19. Ngo VA, Jung JY, Koh JT, et al. Leptin induces odontogenic differentiation and angiogenesis in human dental pulp cells via activation of the mitogen-activated protein kinase signalling pathway. J Endod 2018;44(4):585-591. DOI: 10.1016/j.joen.2017.11.018.
20. Qin W, Gao X, Ma T, et al. Metformin enhances the differentiation of dental pulp cells into odontoblasts by activating AMPK signalling. J Endod 2018;44(4):576-584. DOI: 10.1016/j.joen.2017.11.017.

21. Hu $X$, Zhong $Y$, Kong $Y$, et al. Lineage-specific exosomes promote the odontogenic differentiation of human dental pulp stem cells (DPSCs) through TGF $31 /$ smads signalling pathway via transfer of microRNAs. Stem Cell Res Ther 2019;10(1):170. DOI: 10.1186/s13287019-1278-x.

22. Debeljak M J, Borozan S, Radovanovic A, et al. N-Acetyl-I-cysteine enhances ex-vivo amplification of deciduous teeth dental pulp stem cells. Arch Oral Biol 2016;70:32-38. DOI: 10.1016/ j.archoralbio.2016.06.002.

23. Tu M-G, Ho C-C, Hsu T-T, et al. Mineral trioxide aggregate with mussel-inspired surface nanolayers for stimulating odontogenic differentiation of dental pulp cells. J Endod 2018;44(6):963-970. DOI: 10.1016/j.joen.2018.02.018.

24. Patil VR, Kharat AH, Kulkarni DG, et al. Long-term explant culture for harvesting homogeneous population of human dental pulp stem cells. Cell Biol Int 2018;42(12):1602-1610. DOI: 10.1002/cbin.11065.

25. Onay EO, Yurtcu E, Terzi Y, et al. Odontogenic effects of two calcium silicate-based biomaterials in human dental pulp cells. Adv Clin Exp Med 2018;27(11):1541-1547. DOI: 10.17219/acem/74197.

26. Wang $S, X i a Y, M a T$, et al. Novel metformin-containing resin promotes odontogenic differentiation and mineral synthesis of dental pulp stem cells. Drug Deliv TransI Res 2019;9(1):85-96. DOI: 10.1007/s13346018-00600-3.

27. Atkuri KR, Mantovani JJ, Herzenberg LA, et al. N-acetylcysteine - a safe antidote for cysteine/glutathione deficiency. Curr Opin Pharmacol2007;7(4):355-359. DOI: 10.1016/j.coph.2007.04.005.

28. Berk M, Malhi GS, Gray LJ, et al. The promise of N-acetylcysteine in neuropsychiatry. Trends Pharmacol Sci 2013;34(3):167-177. DOI: 10.1016/j.tips.2013.01.001.

29. Rushworth GF, Megson IL. Existing and potential therapeutic uses for $\mathrm{N}$-acetylcysteine: the need for conversion to intracellular glutathione for antioxidant benefits. Pharmacol Ther 2014;142(2):150-159. DOI: 10.1016/j.pharmthera.2013.09.006.

30. The ocharidou A, Bakopoulou A, Kontonasaki E, et al. Odontogenic differentiation and biomineralization potential of dental pulp stem cells inside Mg-based bioceramic scaffolds under low-level laser treatment. Lasers Med Sci 2017;32(1):201-210. DOI: 10.1007/s10103016-2102-9.

31. Paduano F, Marrelli M, White LJ, et al. Odontogenic differentiation of human dental pulp stem cells on hydrogel scaffolds derived from decellularized bone extracellular matrix and collagen type I. PLoS One 2016;11(2):148-225. DOI: 10.1371/journal.pone.0148225.

32. Soares DG, Rosseto HL, Scheffel DS, et al. Odontogenic differentiation potential of human dental pulp cells cultured on a calciumaluminate enriched chitosan-collagen scaffold. Clin Oral Investig 2017;21(9):2827-2839. DOI: 10.1007/s00784-017-2085-3.

33. Kim Y, Park J-Y, Park H-J, et al. Pentraxin-3 modulates osteogenic/ odontogenic differentiation and migration of human dental pulp stem cells. Int J Mol Sci 2019;20(22):57-78. DOI: 10.3390/ ijms20225778.

34. An S. The emerging role of extracellular $\mathrm{Ca} 2+$ in osteo/odontogenic differentiation and the involvement of intracellular $\mathrm{Ca} 2+$ signalling: from osteoblastic cells to dental pulp cells and odontoblasts. J Cell Physiol 2019;234(3):2169-2193. DOI: 10.1002/jcp.27068. 